\title{
Impact of Coated Ground Plane Edge Diffractions on Amplitude Patterns of Circular Apertures
}

\author{
Nafati A. Aboserwal ${ }^{1}$, Constantine A. Balanis ${ }^{1}$, Life Fellow, IEEE, and Craig R. Birtcher ${ }^{1}$
}

\begin{abstract}
The impact of finite dielectric-covered ground plane edge diffractions on the amplitude patterns of circular apertures is investigated. The model is based on the Geometrical Optics (GO) and the Uniform Theory of Diffraction (UTD) for an impedance wedge. The circular aperture antenna is mounted on square and circular finite ground planes that are coated with a thin lossy dielectric layer. The predictions based on the GO/UTD model are validated by comparisons with experimental results and simulated data.
\end{abstract}

Index Terms-Coated aperture antenna, geometrical optics, diffraction, impedance wedge, surface waves.

\section{INTRODUCTION}

The GTD/UTD [1], [2] has been widely and successfully employed to solve a wide variety of perfectly conducting electromagnetic problems involving diffraction by edges on perfectly conducting surfaces. Its extension to non-perfectly conducting surfaces may provide a significant improvement to the application of ray methods. The non PEC wedge uniform GTD formulation was rigorously derived by asymptotically evaluating the exact solution given by Maliuzhinets [3] using the method of Sommerfeld. It provides a uniform highfrequency solution to the diffraction by a wedge with uniform but different impedances on each face. The asymptotic solution represents the incident, reflected, diffracted, and surfaces wave fields. The surface wave exists over a finite angular range and only for certain surface impedances [4]. The impedance wedge UTD formulation is achieved by introducing suitable multiplying factors. Although these factors involve a special function which is difficult to calculate for a general exterior $(n \pi)$ wedge angle, numerically tractable expressions are available when $n$ is a rational number. In particular, this function can be easily calculated for the four special cases $n=1 / 2,1,3 / 2,2$ [3].

Over many years considerable effort has been devoted to examine aperture antennas mounted in PEC finite/infinite ground planes [5]. However the impact of edge diffractions from a coated finite ground plane on the radiation performance of an aperture antenna has not received adequate attention. This paper addresses this problem using the impedance wedge solution to account for the edge diffractions. For this problem, initially it is assumed that the coated aperture is mounted on an infinite coated ground plane. Then the amplitude patterns of the finite coated ground plane can be modified considerably by the diffractions from the edges, especially in regions of very low field intensity, such as the backward radiation.

1 School of Electrical, Computer and Energy Engineering, Arizona State University, Tempe, AZ 85287-5706. naboserw@asu.edu; balanis@asu.edu; craig.birtcher@asu.edu.

\section{Dielectric-COVERED Aperture Antennas}

For space applications, aperture antennas are covered by a dielectric material to protect them from environmental conditions. The presence of the dielectric cover, however, impacts the radiation characteristics [6], [7]. The spectral domain method was applied to aperture antennas mounted on infinite ground planes [8], and on infinite dielectric-covered ground planes [9]. This method, previously developed to determine the fields radiated from an aperture in a coated cylinder [10], is extended to obtain the solution for the fields radiated by an aperture in a coated ground plane. In particular, the radiation structure considered is an infinite perfectly conducting plane with a circular aperture excited by a specified tangential electric field distribution. The entire plane is generally covered by a thin dielectric layer of complex permittivity, complex permeability, and thickness $t$, as depicted in Fig. 1 .

The fields, produced by the field distribution over the aperture, are found by solving a two-region boundary-value problem. The first region is $0 \leq z \leq t$ and the second region is $t \leq z \leq \infty$. The fields inside the waveguide are uniquely defined by a discrete TE vector potential. The fields inside the dielectric coating are represented by standing waves and are described by integral transforms of TE and TM potentials [9]. The boundary conditions at the aperture, and at the outer surface of the dielectric coating, are used to solve for the unknowns. For the same aperture excitation, the far fields radiated by a coated aperture are related to those of an uncoated by simple multiplicative functions which depend on the parameters of the dielectric coating and the off-axis angle $(\theta)$.

$$
\begin{aligned}
& E_{\theta}(r, \theta, \phi)=f(\theta) E_{\theta}^{0}(r, \theta, \phi) \\
& E_{\phi}(r, \theta, \phi)=g(\theta) E_{\phi}^{0}(r, \theta, \phi)
\end{aligned}
$$

where $E_{\theta}$ and $E_{\phi}$ are the electric field components of the covered aperture, and $E_{\theta}^{0}$ and $E_{\phi}^{0}$ are the electric field components of the uncovered aperture. For the definitions of $f(\theta)$ and $g(\theta)$ functions, refer to [8] and [9].

When the permittivity of the dielectric layer equals the permittivity of free space, or when the thickness of the dielectric coating $t$ is zero, (1) and (2) reduce to those for the uncoated case.

The antenna under consideration consists of a circular waveguide, opening onto a finite, perfectly electric conducting flat ground plane coated with a thin dielectric layer of thickness $t$, and complex permittivity and permeability, as shown in Fig. 1. The circular waveguide dominant $\mathrm{TE}_{11}$ mode is assumed over the circular aperture, and the higher order reflected modes are assumed to be negligible. 


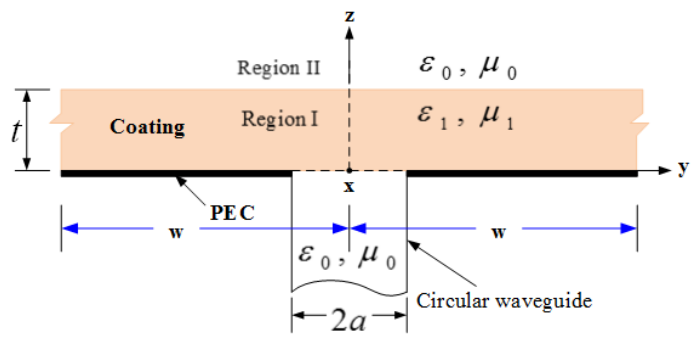

Fig. 1. Two-dimensional geometry of a circular aperture antenna mounted on a coated perfectly conducting plane.

\section{IMPEDANCE SURFACE BOUNDARY CONDITIONS}

The increasing application of lossy/lossless dielectric materials increases the need for suitable methods to characterize the response of these materials, including the scattering effects of edge/wedges and discontinuities. The approximate impedance boundary conditions are an effective approach to model the surface impedance. This model is good for conductor and lossy materials where there is some penetration of the field. The impedance surface boundary conditions, introduced by Leontovich in 1940's [11], are widely used to simulate the material properties of a scatterer; they can be very helpful in simplifying the analytical and numerical solutions of scattering problems. The surface impedance concept is a popular and efficient tool in computational electromagnetics. It has been widely used to analyze electromagnetic problems for which the material properties or surface characteristics are important. The impedance boundary condition is a useful approximation for many physical problems because it includes the material properties without explicitly involving the fields within the material. It represents approximate relations between the electromagnetic fields and the quantities at the surface of the conductor, so that the conducting region does not need to be included in the mesh and can be represented by surface impedance boundary conditions (SIBCs) [12], [13].

The normalized equivalent surface impedance may be obtained from a simple transmission line model [14]. By considering the coating as a section of a transmission line of length $t$ and normalized characteristic impedance $\eta=\sqrt{\mu_{1} \varepsilon_{0} / \mu_{0} \varepsilon_{1}}$, terminated with the zero impedance of the PEC, the input impedance, corresponding to the normalized equivalent surface impedance of a obliquely incident plane wave, is

$$
\eta_{e q}=j \eta \tan \left(k_{1} t \sin \phi_{t}\right) \quad \sin \phi_{t}=\sqrt{1-\frac{\mu_{0} \varepsilon_{0}}{\mu_{1} \varepsilon_{1}} \cos ^{2} \phi_{i}}
$$

where $k_{1}=\omega \sqrt{\mu_{1} \varepsilon_{1}}$ is the phase constant.

\section{DIFFRACTED FIELDS}

The two-dimensional geometry of aperture antennas mounted on square/circular coated ground planes is shown in Fig. 1. Both the square and circular ground planes are treated similarly. Far-zone E- and H-plane amplitude patterns are analytically calculated for ground planes covered with a dielectric layer following the procedure described in [4]. To examine the impact of the dielectric-covered ground plane geometry on the E- and H-plane amplitude patterns, a comparison of the square and circular ground planes, where the side of the square is equal to the diameter of the circular, is carried out.

The incident field at points $Q_{d 1}$ and $Q_{d 2}$, as shown in Fig. 2 , is found from (1)-(2) after substituting $\theta=\pi / 2$ and $r=\mathrm{w}$, where $\mathrm{w}$ is the half width of the square ground plane or the radius of the circular ground plane, and $\rho_{c 1}$ and $\rho_{c 2}$ are the distances between the caustic at the diffraction points $\left(Q_{d 1}\right.$ and $Q_{d 2}$ ) and second caustic of the diffracted ray [4].

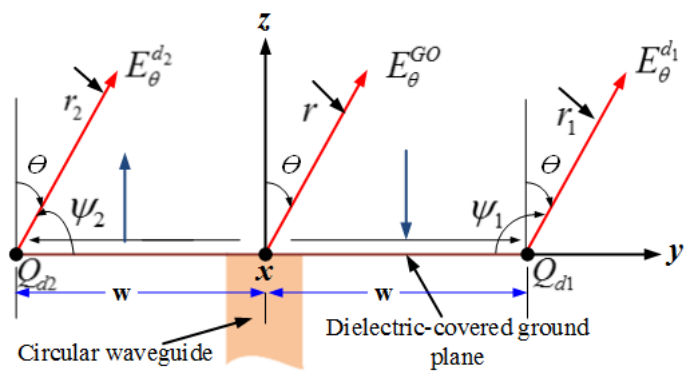

Fig. 2. Diffraction mechanism by edges of dielectric-covered ground planes.

For the far-field observations, the field diffracted from the diffracting points $Q_{d 1}$ and $Q_{d 2}$, for either the square or the circular ground plane, after suppressing the spatial variation factor $\left(e^{-j k r} / r\right)$, can be written as

$$
\begin{aligned}
& E_{\theta}^{d_{1}}=E_{\theta}^{i}\left(Q_{d 1}\right) D^{h}\left(L_{1}, \psi_{1}, 0, \frac{\pi}{2}, 2\right) \sqrt{\rho_{c 1}} e^{+j k \mathrm{w} \sin \theta} \\
& E_{\theta}^{d_{2}}=E_{\theta}^{i}\left(Q_{d 2}\right) D^{h}\left(L_{2}, \psi_{2}, 0, \frac{\pi}{2}, 2\right) \sqrt{\rho_{c 2}} e^{-j k \mathrm{w} \sin \theta}
\end{aligned}
$$

for the far-zone E plane, and

$$
\begin{aligned}
& E_{\phi}^{d_{1}}=E_{\phi}^{i}\left(Q_{d 1}\right) D^{s}\left(L_{1}, \psi_{1}, 0, \frac{\pi}{2}, 2\right) \sqrt{\rho_{c 1}} e^{+j k \mathrm{w} \sin \theta} \\
& E_{\phi}^{d_{2}}=E_{\phi}^{i}\left(Q_{d 2}\right) D^{s}\left(L_{2}, \psi_{2}, 0, \frac{\pi}{2}, 2\right) \sqrt{\rho_{c 2}} e^{-j k \mathrm{w} \sin \theta}
\end{aligned}
$$

for the far-zone $\mathrm{H}$ plane; for the definitions of $L_{1}, \psi_{1}, \rho_{c 1}$, $L_{2}, \psi_{2}$, and $\rho_{c 2}$, refer to [15].

$D^{s}$ and $D^{h}$ are, respectively, the soft and hard polarization impedance wedge diffraction coefficients [16] which depend implicitly on the surface impedances modelling of the wedge faces [4]. Because these coefficients were derived assuming that the antenna was mounted on the lower face of the wedge, all angles in this paper must be referenced to the underside face of the wedge. Because the incident field for the H-plane vanishes at the grazing angle, the rate of change of the incident field at the point of diffraction is used [4].

For the circular ground plane, the circular edge behaves as a continuous ring radiator [4], which leads to the formation of a caustic where the diffracted field are infinite. Therefore, a caustic correction is needed for angles at and near the axis of the antenna. Using the method of equivalent currents and wedge diffraction coefficients, the caustic problem can be corrected [4]. In this method, equivalent magnetic and electric currents are created along the edge of the ground plane. Then radiation integrals are used to obtain the fields due to these currents, which correct the diffracted fields at and near the symmetry axis of the antenna. 
For the square ground plane, the edge diffractions do not contribute significantly to the $\mathrm{H}$-plane radiation pattern in the backlobe region. However, the edge diffractions in the E-plane are much more intense and contribute more significantly to the overall pattern, above and below the ground plane. Therefore, one needs to include the contributions from the E-plane edge diffractions using the method of equivalent currents [4].

The surface wave components, and their corresponding surface wave transition fields, are necessary to provide continuity of the fields, even in a smaller angular region where they exist; their formulas are given in Chapter 14 of [4].

\section{PATtern VAlidation}

A model for the circular and square dielectric-covered ground planes with the circular waveguide mounted at the center has been constructed. The aperture antenna is assumed to be exicted by the $\mathrm{TE}_{11}$-mode circular waveguide. The width of the coated square ground plane and the diameter of the coated circular ground plane are $30.48 \mathrm{~cm}$. The relative permittivity $\left(\varepsilon_{r}\right)$ and the loss tangent $(\tan \delta)$ of the coating material are, respectively, 2.9 and 0.02 at $10 \mathrm{GHz}$. The thickness of the dielectric layer, made from polycarbonate (Lexan), is $0.25 \mathrm{~cm}$ and the normalized surface impedance is $0.7480+j 0.4894$ calculated using (3). The validity of the radiation pattern formulation over the main beam and the near and far sidelobes has been verified by calculating the farzone E- and H-plane amplitude patterns of the aperture. The frequency at which the measurements were performed is 10 GHz. The diameter of the aperture is $2.38 \mathrm{~cm}$. Measurements and numerical data based on diffraction techniques were also compared with the HFSS simulations.

Figs. 3-4 exhibit the far-zone E- and H-plane amplitude patterns of a circular aperture mounted, respectively, on circular and square dielectric-covered ground planes. Because the amplitude patterns of interest are in the principle planes, the most significant diffractions come from two diffraction points which are diametrically opposite to each other and along the principle planes [4]. Good agreement between GO/UTD calculations, experiments, and HFSS simulations is indicated. The amplitude patterns in the E-plane are more broad than those in the H-plane as shown in Figs. 3-4. The ripples in the amplitude patterns, especially in the backlobe region, are attributed to the impedance edge diffractions. The ripples shown in the patterns are due of the constructive and destructive interference of the diffractions from the diametrically opposite diffraction points. In the E-plane, these ripples are more significant because the incident electric field at the point of diffraction is more intense in this plane than in the H-plane.

In addition, the amplitude patterns of the same antennas without coating in the $\mathrm{E}$ and $\mathrm{H}$ planes [15] are compared to those obtained for the coated case. It can be seen that the amplitude patterns of the coated ground planes have stronger radiation especially in the back and far sidelobes because of the existence of surface waves. The ripples in the forward lobe of the coated-antenna patterns are more severe because the edge excitations are very strong to have a significant effect on the overall pattern.
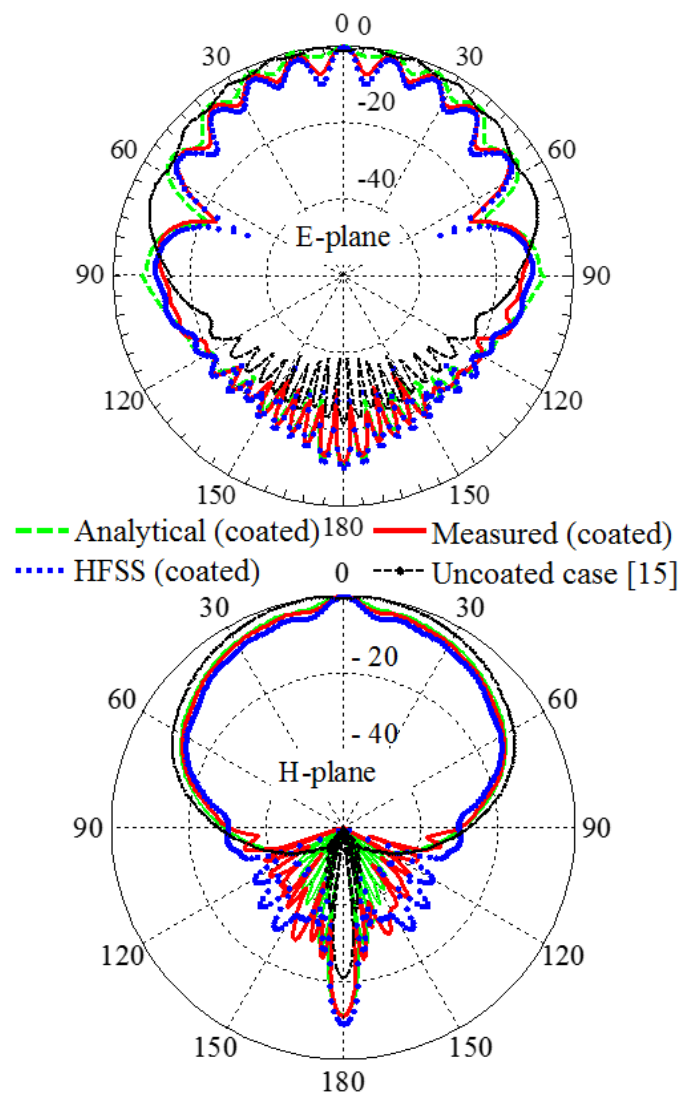

Fig. 3. Far-zone E- and H-plane amplitude radiation patterns of a circular aperture antenna mounted on a circular coated ground plane at $10 \mathrm{GHz}$.
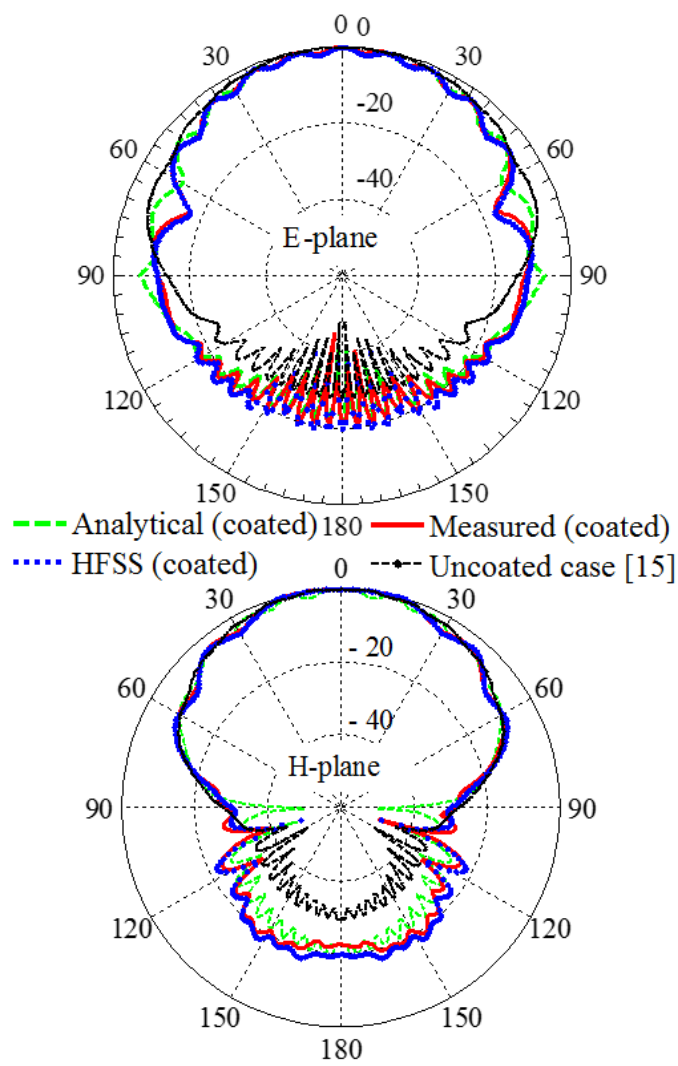

Fig. 4. Far-zone E- and H-plane amplitude radiation patterns of a circular aperture antenna mounted on a square coated ground plane at $10 \mathrm{GHz}$. 
Although the side of the square is equal to the diameter of the circular, it is clear from the patterns of Figs. 3,4 that the E- and H-plane amplitude patterns of the circular ground plane are greater than those of the square ground plane at and near the antenna axis $\left(\theta=180^{\circ}\right)$. These are due to the ring radiator [4] which contributes about an additional 8-10 dB.
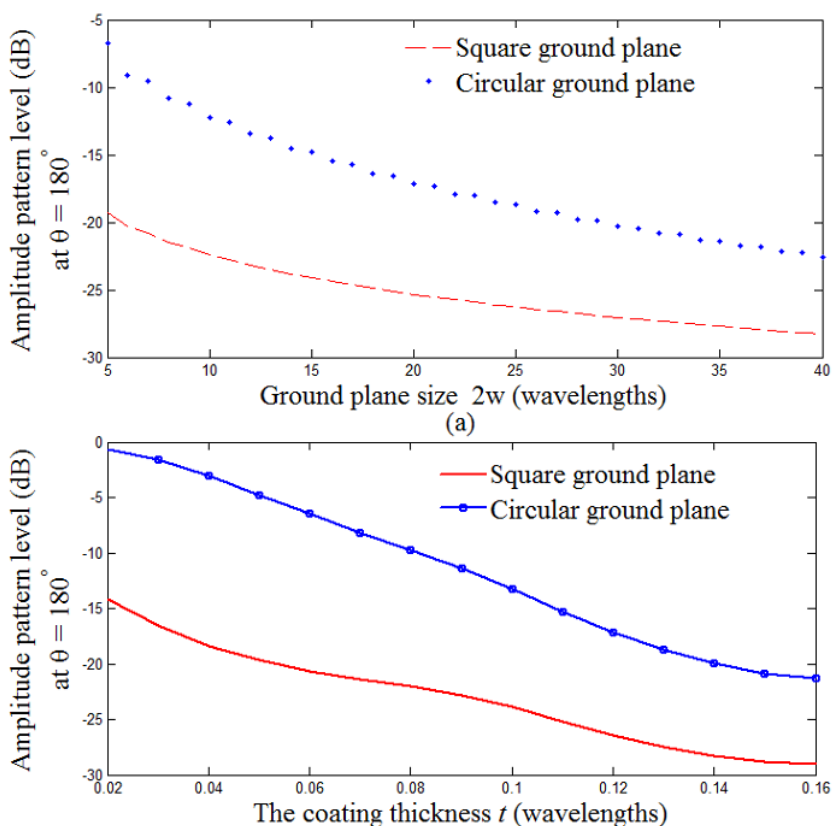

(b)

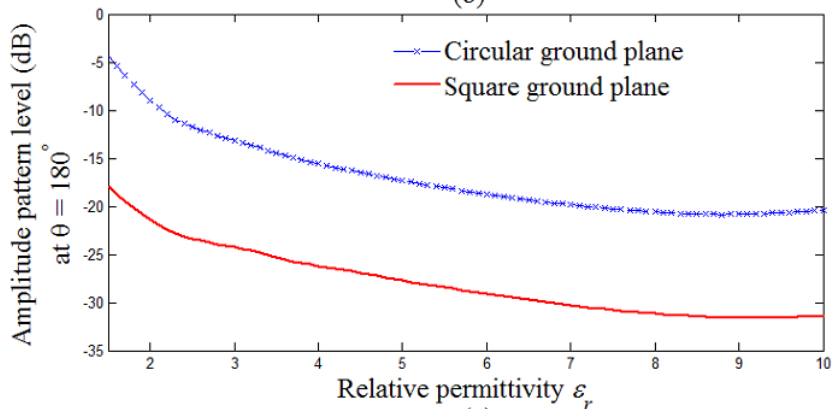

(c)

Fig. 5. Amplitude pattern level at $\theta=180^{\circ}$ due to: (a) size of ground plane, (b) coating thickness, and (c) relative permittivity.

The radiation amplitude in the back region of the aperture antenna mounted on coated ground planes depends significantly on the size and geometry of the ground plane. Fig. 5(a) indicates, as expected, that the amplitude pattern level at $\theta=180^{\circ}$ decreases monotonically with increasing the ground plane size for both geometries, circular and square. Because of the ring radiator of the circular ground plane, its amplitude in the back lobe is more than that for the square ground plane. As the size increases, the edge diffractions do not introduce any noticeable ripples in the forward region, and the diffraction level in the back region becomes very comparable to the noise level in the detection system. The periodicity of the ripples in the patterns depends upon the size of the ground plane, while the amplitude of the ripples depends upon the strength of the edge excitation.

The amplitude pattern level at $\theta=180^{\circ}$ for different coating thickness is also displayed in Fig. 5(b) for circular and square coated ground planes. It is apparent that the back lobe radiation at $\theta=180^{\circ}$ is stronger for thinner coatings. As shown in Fig. $5(\mathrm{c})$, by increasing the relative permittivity of the coating, the amplitude level of the diffracted field at $\theta=180^{\circ}$ decreases for both geometries. Based on the simulated data, increasing either the thickness or relative permittivity of the dielectric coating leads to less radiation in the back lobe region.

\section{CONCLUSIONS}

The impact of the finite dielectric-covered ground plane edge diffractions on the amplitude patterns of a circular aperture is investigated. The diffraction of electromagnetic waves for impedance wedges (half plane with two face impedances for our case) is investigated. The UTD diffracted fields and the GO fields for a dielectric-covered PEC plane are vectorially combined to determine the far-zone amplitude patterns in the E- and H-planes. The study indicates that the finite coated ground plane edge diffractions do impact the main forward lobe pattern, especially for the E-plane. Their most significant contribution appears in far side and back lobes. All these results are indicators that the finite edge contributors must be considered to obtain more accurate amplitude radiation patterns. Moreover, the impact of the ground plane size, the coating thickness, and the relative permittivity has been investigated in this work.

\section{REFERENCES}

[1] J. B. Keller, "Geometrical Theory of Diffraction," J. Opt. Soc. Amer, Vol. 52, pp. 116-130, February 1962.

[2] R. G. Kouyoumjian and P. H. Pathak, "A Uniform Geometrical Theory of Diffraction for an Edge in a Perfectly Conducting Surface," Proceedings of the IEEE, Vol. 62, No. 11, pp. 1448-1461, November 1974.

[3] G. D. Maliuzhinets, "Excitation, Reflection and Emission of Surface Waves from a Wedge with Given Faces Impedances," Sov. Phys. Doke., Vol. 3, pp. 752-755, March 1958.

[4] C. A. Balanis, Advanced Engineering Electromagnetics, 2nd ed. : John Wiley and Sons, NJ, 2012.

[5] C. A. Balanis, "Pattern Distortion Due to Edge Diffraction," IEEE Trans. Antennas Propagat., Vol. 18, No. 4, pp. 561-563, July 1970.

[6] A. K. Bhattacharyya and D. L. Sengupta, Radar Cross Section Analysis and Control, 3rd ed. : Artech House, MA, 1991.

[7] M. D. Deshpande and P. D. Prabhakar, "Analysis of Dielectric Covered Infinite Array of Rectangular Microstrip Antennas," IEEE Trans. Antennas Propagat., Vol. 35, No. 6, pp. 732-736, June 1987.

[8] C. A. Balanis, Antenna Theory: Analysis and Design, 3rd ed. : John Wiley and Sons, NJ, 2005.

[9] C. M. Knop and G. I. Cohn, "Radiation from an Aperture in a Coated Plane," Radio Sci. J. Res., Vol. 68D, No. 4, pp. 363-378, April 1964.

[10] J. Wait and W. Mientka, "Calculated Patterns of Slotted Elliptic-Cylinder Antennas," Appl Sci. Res., Vol. 7, No. 1, pp. 449-462, December 1959.

[11] M. A. Leontovich, "Approximate Boundary Conditions for the Electromagnetic Field on the Surface of a Good Conductor, Investigation of Propagation of Radio Waves, Part III," Academy of Sciences, pp. 5-12, Moscow, 1948.

[12] T. B. A. Senior and J. L. Volakis, "Approximate Boundary Conditions in Electromagnetics," IEEE Press, U.K., 1995.

[13] T. Griesser, "High-frequency Electromagnetic Scattering from Imperfectly Conducting Structures," Ph.D. dissertation, Arizona State University, Tempe, August 1988.

[14] R. Collin, Foundations for Microwave Engineering, 2nd ed. : McGrawHill, NY, 1992.

[15] N. Aboserwal, C. Balanis, and C. Birtcher, "Impact of Finite Ground Plane Edge Diffractions on Radiation Patterns of Aperture Antennas," Progress In Electromagnetics Research B, Vol. 55, pp. 1-21, 2013.

[16] R. Tiberio, G. Pelosi, and G. Manara, "A Uniform GTD Formulation for the Diffraction by a Wedge with Impedance Faces," IEEE Trans. Antennas Propagat., Vol. 33, No. 8, pp. 867-873, August 1985. 